\title{
Analysis of Grain-Resolved Data from Three-Dimensional X-Ray Diffraction Microscopy in the Elastic and Plastic Regimes
}

Juul, Nicolai Ytterdal; Oddershede, Jette; Winther, Grethe

Published in:

J O M

Link to article, DOI:

$10.1007 / \mathrm{s} 11837-019-03829-6$

Publication date:

2019

Document Version

Peer reviewed version

Link back to DTU Orbit

Citation (APA):

Juul, N. Y., Oddershede, J., \& Winther, G. (2019). Analysis of Grain-Resolved Data from Three-Dimensional XRay Diffraction Microscopy in the Elastic and Plastic Regimes. J O M, 72, 83-90.

https://doi.org/10.1007/s11837-019-03829-6

\section{General rights}

Copyright and moral rights for the publications made accessible in the public portal are retained by the authors and/or other copyright owners and it is a condition of accessing publications that users recognise and abide by the legal requirements associated with these rights.

- Users may download and print one copy of any publication from the public portal for the purpose of private study or research.

- You may not further distribute the material or use it for any profit-making activity or commercial gain

- You may freely distribute the URL identifying the publication in the public portal 


\title{
Analysis of grain-resolved data from three-dimensional X-ray diffraction microscopy in the elastic and plastic regimes
}

\author{
Nicolai Ytterdal Juul ${ }^{\mathrm{a}}$, Jette Oddershede ${ }^{\mathrm{b}}$ and Grethe Winther ${ }^{\mathrm{a}^{*}}$ \\ a Department of Mechanical Engineering, Technical University of Denmark, Produktionstorvet \\ 425, 2800 Kgs. Lyngby, Denmark

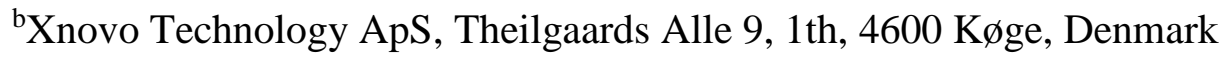

\begin{abstract}
:
The combination of experimental data and polycrystal plasticity modelling is a strong tool to advance our understanding of the behaviour of individual grains and how these interact. The present paper illustrates how much information a single three-dimensional X-ray diffraction experiment contains and how this may be analysed and compared to model predictions. The data covers the elastic and plastic regimes (0.1, 1 and 5\% strain) of a tensile experiment on a fully recrystallized stainless steel sample. The number of bulk grains analysed is of the order of 200 . Specific phenomena considered are stress correlations across twin boundaries, stress states and resolved shear stresses in the elastic and plastic regimes along with lattice rotations.
\end{abstract}

\section{Introduction}

The emergence of the synchrotron-based technique of three-dimensional X-ray diffraction (3DXRD) [1], also known as high-energy diffraction microscopy (HEDM) [2], has opened up new routes to monitor microstructural phenomena in polycrystalline materials. The three-dimensional character of the data combined with the penetration power of high-energy X-rays allows mapping of metallic microstructures embedded in the bulk of the sample and thus unaffected by potential effects of a free sample surface. Due the non-destructive character of the technique the microstructural response to external stimuli, e.g. deformation or temperature changes, may be monitored in-situ. Typical parameters recorded are crystallographic orientations and elastic strains. A wide range of different variants of the technique has been developed [3-9], in particular enabling different trade-offs between the spatial and temporal resolution of the obtained data, which must also be balanced towards the measurement time.

To broaden the scope of 3DXRD experiments beyond the specific sample volume investigated, it is important that analysis of the experimental data is combined with modelling and simulation of the microstructural evolution. In this way the assumptions underlying the models can be tested, key microstructural parameters for various material behaviours may be identified, and the accuracy of the simulated microstructural evolution can be evaluated. The final goal is of course to establish new models with improved accuracy, which will enable prediction of material performance also in other materials and upon enforcement of more complex loading conditions than typically allowed in these challenging experiment. 
The present paper presents the results obtained from a single experiment conducted at the Cornell High Energy Synchrotron Source on an austenitic stainless steel sample deformed in tension to 5\% elongation. The focus of the paper is on the exploitation of the data in crystal plasticity modelling and simulations. A spatially resolved grain map was obtained in the undeformed state and serves as input to further analysis and simulations. In the deformed state, grain-averaged full strain tensors and crystallographic orientations are extracted from the diffraction data. The wealth of information that may be extracted from a single data set is exemplified through analysis of the elastic grain interaction across grain and twin boundaries, the grain orientation dependence of the grainresolved stress states during plastic deformation, and finally the rotation and subdivision of individual grains.

\section{Experimental}

The sample was a fully recrystallized austenitic $316 \mathrm{~L}$ stainless steel annealed at $1100^{\circ} \mathrm{C}$ for 65 minutes to obtain an average grain diameter of $70 \mu \mathrm{m}$. The 3DXRD experiment was conducted at beamline F2 at the Cornell High Energy Synchrotron Source (CHESS). In the undeformed state both a near-field (QImaging Retiga 4000DC) and a far-field detector (GE Revolution 41RT [10]) were employed. Data from the near-field detector were analysed to reconstruct a grain map with high spatial resolution (Fig. 1a). In the deformed state only the far-field detector was used for data on grain orientations, elastic strains and stresses. The sample was illuminated with a $0.10 \mathrm{~mm}$ high box beam covering the entire cross section of the sample $\left(0.7 \times 0.7 \mathrm{~mm}^{2}\right)$. The load frame RAMS2 (Rotation and Axial Motion System, $2^{\text {nd }}$ build) [11] was used to load the sample in uniaxial tension while simultaneously allowing illumination of the sample over a rotation range of $360^{\circ}$ in $\omega$ around the tensile axis. In total five layers along the tensile direction were scanned. Data were recorded at $0,0.1,1,3$ and $5 \%$ sample elongation.

The diffraction data were analysed using the FABLE software package [12]. The initial indexing of the diffraction spots to individual grains was conducted with GrainSpotter [13] for the undeformed state of the sample. The centre of mass (CMS) positions, orientations, relative volumes and elastic strains of individual grains at all load steps were fitted using FitAllB [14]. Fig. $1 \mathrm{~b}$ shows the elastic tensile strain of each grain in the five layers at $1 \%$ elongation. In order to have a coherent map corresponding to the illuminated volume, layers were merged into one map by comparing centre of mass and orientation of each grain segment in the neighbouring layers using distance and orientation criteria of $100 \mu \mathrm{m}$ and $0.2^{\circ}$ at 0 and $0.1 \%$ elongation and $120 \mu \mathrm{m}$ and $2.7^{\circ}$ at 1 and $5 \%$ elongation. Orientation and stress data were recalculated as the volume weighted averages of grain parts in different layers. More details on the experiment and reconstruction of grain maps may be found in [15].

The measured elastic strain tensors were converted to stress using the elastic constants: $c_{11}=206$ $\mathrm{MPa}, c_{12}=133 \mathrm{MPa}$, and $c_{44}=119 \mathrm{MPa}$ [16]. Table I states the uncertaintiesof individual components of the stress tensor in the coordinate system of the macroscopic tensile deformation averaged over all grains for 0.1 and $1 \%$ elongation. These values are of the order of $10 \%$ compared with the macroscopic tensile stress of 167 and $197 \mathrm{MPa}$ at the two levels of elongation. The fact that this is the level for all stress components ensures high accuracy also after rotation of the stress tensor to other coordinate systems. In all such rotations in the subsequent sections, the uncertainties are recalculated (see [17] for details). 


\section{Results}

High-resolution spatial data in the undeformed state combined with the CMS data of each grain provides the possibility to study the interaction between neighbouring grains in the sample across boundaries that are well characterised. The accuracy of the stress tensor allows analysis of stress states in crystallographic coordinate systems rather than the macroscopic tensile coordinates. In the present case, these features of the data were exploited to analyse the interaction across the boundaries of annealing twins in the elastic regime compared to the interaction across ordinary grain boundaries. In addition, the measured stress states in the plastic regime as well as calculations of the resolved shear stress on the individual slip systems were compared to predictions of classical polycrystal plasticity models. Finally, crystal plasticity predictions of the lattice rotations are compared to the experimentally determined rotation paths from 0 to $5 \%$ elongation, including discussions on grain subdivision.

\subsection{Grain interactions in the elastic regime}

In the face-centred cubic unit cell the stiffest direction is $<111>$ and the most compliant is $<100>$. Elastic interactions involving these two directions may therefore be expected to represent extreme situations. As the $<111>$ direction is also the normal to twin boundaries, the stiffest direction is common to the two grain volumes separated by the twin boundary. Annealing twin boundaries are therefore selected for this study of elastic grain interactions. Boundary planes were identified as the best fitted plane through all of the voxels in the grain map obtained with the near field detector connecting two neighbouring grains. The boundary plane and the crystallographic misorientation between neighbouring grains were used to identify twins. Fig. 2 illustrates two grains with twins.

As the crystallographic misorientation between twins is a $60^{\circ}$ rotation around the twin boundary normal, the selected twin pairs are further characterized by having the tensile axis of one part of the twin pair aligned close to the elastically most compliant $<100>$ direction while the direction of the tensile axis of the other part is significantly stiffer. The orientations of the tensile axes of the two parts of the selected twin pairs are presented in Fig. 3a. It should be noted that a direct consequence of the crystallography of this selection is that the inclination of the twinning plane to the tensile axis of the experiment is restricted to a range of $45-65^{\circ}$, ensuring not only crystallographically similar boundaries, but also boundaries with a preferred orientation with respect to the applied tensile deformation as is also the case in the two grains in Fig. 2.

The stress directions along the tensile axis and normal to the twin boundary are illustrated in Fig. 2a. These stress components are calculated from the average elastic strain tensor in each part of the twin pair. The stress along the tensile direction at $0.1 \%$ elongation correlates with crystallography as expected; the stress in the twin parts with the tensile axis close to $<100>$ is lower than in the stiffer twin part, although with significant scatter [15]. To investigate the correlation across the twin boundary, the stress components normal to the twin boundary of the compliant and stiff parts are plotted against each other in Fig. 3b. For about half of the 20 twin pairs, the stress values cluster in the range 0-100 MPa. However, in the rest of the twin pairs larger 
stresses are observed in either the compliant or the stiff part. A weighted correlation coefficient of $r^{2}=0.03$ demonstrates the absence of a special correlation across the twin boundary normal, even though this is a stiff direction common to both twin parts. This also means that twin boundaries are not special in this respect and may be treated like conventional boundaries.

\subsection{Stress states in the plastic regime}

The high accuracy of the determined strain also allows calcuation of the resolved shear stress on the individual slip systems in each grain. Comparison of the data at 0.1 and $1 \%$ elongation further allows analysis of the development from the elastic to the fully plastic state. For clarity, the focus is on grains with the tensile axes aligned with the corners of the stereographic triangle $(<100>$, $<111>,<110>$ ) and with a direction in the middle of the triangle $(<321>)$. The resolved shear stresses on the 12 fcc slip systems listed in Table II are presented in Fig. 4. It is noted that all grain orientations have been converted to the stereographic triangle where slip system -b2 has the highest theoretical Schmid factor in uniaxial tension. The figure presents the mean values for a number of grains in the immediate vicinity of the ideal orientations. Examples for individual grains may be found in [17].

In the elastic regime at $0.1 \%$ elongation, the upper row in Fig. 4 demonstrates that the number of highly stressed slip systems in the $<100>$ orientation is eight with essentially the same stress on all of these. This is in agreement with the expected Schmid factors calculated for uniaxial tension. Near $<111>$ six systems have high shear stresses, as also expected from the Schmid factors. It is, however, clear that systems b2 and b1 have higher stresses than the others. This is in agreement with the fact that the exact orientation of the seven grains considered are closer to the $<110>$ $<111>$ line of the triangle than to the ideal $<111>$ orientation. For the $<110>$ oriented grains, four systems have almost the same resolved shear stress, which is about twice the value of other slip systems. The occurrence of the four dominant systems is in full agreement with the Schmid factors. Finally, the grains near $<321>$ in the middle of the triangle exhibit a fairly large variation in the magnitude of the resolved shear stress. This variation is also as expected based on the Schmid factors, which also agree with the finding of the highest stress value for system b2. For the ideal $<321>$ the second highest shear stress value on systems b1 and a2, should be $75 \%$ of the maximum. These two systems are also those with the second highest measured values, although system b1 is almost as stressed as b2. Summing up, the measured resolved shear stress on the slip systems agrees well with the Schmid factors calculated for an applied uniaxial stress along the tensile direction.

In the plastic regime at $1 \%$ elongation, some of the stress states have evolved. Similar changes in stress state in the plastic regime have also been observed in other studies, e.g. [18]. According to the classical polycrystal plasticity model of Bishop and Hill [19], the grains should now be in one of 56 stress states. By means of symmetry this number reduces to five, which are all expected to be represented in uniaxial tension. Each theoretical state has the same non-zero resolved shear stress on either six or eight systems. The resolved shear stress on the remaining systems is zero [20]. The slip systems expected to have the high values for the selected grain orientations are marked by the colours in the lower row of Fig. 4. In this context it is noted that none of the resolved shear stress values reaches the expected critical value. This is attributed to the observed relaxation of $20 \%$ of the tensile stress immediately after loading. Relaxation during data acquisition were negligible. As evident from the lower row of Fig. 4, the stress state of the grains near $<100>$ has 
not changed qualitatively from 0.1 to $1 \%$ elongation. Only the stress level has increased as one should expect. This is according to expectation as the predicted Bishop-Hill stress state for $<100>$ is pure uniaxial tension along the tensile axis. The same is the case for the ideal $<111\rangle$ orientation. Fig. 4 reveals that the six highly stressed systems from $0.1 \%$ are still the same, but at $1 \%$ they are equally stressed. This indicates that the stress state has rotated slightly away from uniaxial stress along the actual crystallographic direction of the grains to get all grains in the Bishop-Hill stress state, corresponding to uniaxial stress along the $<111>$ direction. By contrast, the expected BishopHill stress state for the grains near $<110>$ is not achieved. Although the eight systems in the expected state are all highly stressed, their stress levels are not the same. The four systems with high shear stresses at $0.1 \%$ are still the ones with the highest values, although the stress on the other four systems are higher than at $0.1 \%$. Inspection of the stress states of individual grains reveals that some are in the Bishop-Hill state, some are in the applied uniaxial state and some lie in between [17]. For the grains near the $<321>$ orientation the average stress state is qualitatively similar to that at $0.1 \%$ with large variations in the stress level of the individual slip systems. Inspection of individual grains reveals a dominance of the stress state indicated by the grey colour in Fig. 4 but also a number of grains in the expected Bishop-Hill stress states of the $<111>$ and $<110>$ orientations [17]. The theoretically expected Bishop-Hill stress state of the $<321>$ orientation is the one corresponding to the $<110>$ orientation. The finding of another dominant state therefore indicates inadequacies of the model.

In order to conduct a more detailed analysis of the orientaiton dependence of the stress states in relation to the Bishop-Hill predictions, the Lode parameter was employed. The Lode parameter takes different definitions. Here the following is employed:

$$
\text { Lode }=\operatorname{asin}\left(\frac{J_{3}}{2}\left(\frac{3}{J_{2}}\right)^{1.5}\right) / 3
$$

Equation 1

where

$$
J_{2}=\frac{1}{6}\left[\left(\sigma^{\prime}{ }_{11}-\sigma_{22}^{\prime}\right)^{2}+\left({\sigma^{\prime}}_{11}-\sigma_{33}^{\prime}\right)^{2}+\left({\sigma^{\prime}}_{22}-{\sigma^{\prime}}_{33}\right)^{2}\right]+\left({\sigma^{\prime}}_{12}{ }^{2}+{\sigma^{\prime}}_{13}{ }^{2}+{\sigma^{\prime}}_{23}{ }^{2}\right)
$$

and

$$
J_{3}=\operatorname{det}\left(\sigma^{\prime}\right)
$$

Equation 2

are the second and third invariants of the deviatoric stress tensor, $\sigma^{\prime}$.

The advantage of invoking the Lode angle lies in the fact that the stress tensor for each BishopHill state reduces to one number. The Lode angles for uniaxial tension and plane stress are $\pi / 6 \approx 0.52$ and 0 , respectively. The background colours of the stereographic triangles in Fig. 5 represent the Lode angles for the theoretical Bishop-Hill states. Uniaxial tension is found near $<100>$ and $<111>$ whereas plane stress conditions apply further from $<100>$ and near $<110>$. It is seen that the Lode angles for the measured stress states at $0.1 \%$ elongation (Fig. $5 \mathrm{a}$ ) in general are higher than at $1 \%$ elongation (Fig. 5b), in particular near $<110>$. This demonstrates that the stress states in the elastic regime are closer to the applied uniaxial state, while they are closer to the 
Bishop-Hill states in the plastic regime. This is in agreement with the conclusions for the resolved shear stresses on the slip systems.

The $<321>$ orientation lies in the region coloured dark blue, which extends into the triangle from the $<110>$ corner, but the $<321>$ orientation is also close to the border where the dark red and turquoise regions meet. In Fig. 5b, it is seen that the Lode angle is higher than expected near $<321>$. The stress state with a Lode angle near 0.17 (turquoise) therefore extends further into the triangle than originally predicted by the Bishop-Hill model. It is also evident from the figure that the uniaxial stress state extends further into the triangle from the $<100>$ corner than expected.

Polycrystal plasticity calculations reveal that the origin of the deviations of the expected orientation dependence of stress state lies in a lowering of the plastic work, when grains near $<321>$ and $<100>$ orientations do not deform with an axisymmetric tensile strain as normally assumed in the Bishop-Hill model [17]. Finite element based crystal plasticity simulations using the experimentally determined grain map as input reproduce the grain orientation dependence of the measured stress states well. In agreement with the experiment only about half of the grains were determined to be in a Bishop-Hill stress state, although the agreement between experiment and prediction at the level of individual grains was poor [17,21]

\subsection{Lattice rotations}

Fig. 6a shows the experimentally measured rotation of the crystallographic tensile axis for all nonsurface grains from 0 to $5 \%$ elongation. The corresponding rotation paths are predicted by crystal plasticity simulations using the experimentally determined grain map in Fig. 1a as input. The predictions are made using the WARP3D polycrystal plasticity code with isotropic Voce hardening [22]. The overall trend for rotation towards $<111>$ and $<100>$ are reproduced by the model. However, the rotation rate is greatly underestimated by the model, and the experimental data also exhibit a larger variation in the rotation direction. This is in agreement with general observations $[6,21,23]$. Analysis of the lattice rotations and the origin of the inadequacy of the model predictions is pending.

As plastic deformation occurs orientation gradients develop within each grain, leading to broadening of the diffraction spots. The present experiment does not have the resolution to analyse this in detail. Nevertheless, the directionality of the spot broadening can be associated with gradients in the activity of individual slip systems [24,25], as illustrated in Fig. 7. The spot is from a grain near $<100>$ and the slip systems giving rise to the orientation spread are among those expected based on the resolved shear stresses for grains of this orientation (see Fig. 4). The slip systems have been converted to their corresponding notation for grain orientations in the same stereographic triangle as for Fig. 4.

\section{Discussion}

The 3DXRD method has provided new possibilities to observe the dynamics of individual grains deeply embedded in a polycrystal during elastic and plastic deformation. The design of stress rigs that fit into the limited space available, allows rotation to obtain diffraction from different angles and provide sufficient mechanical stability has also been crucial for the success of the technique 
within plastic deformation. The present paper focuses on a demonstration of the wealth of information a single experiment may provide.

The present experimental set up with two detectors placed at different distances from the sample allows for good spatial resolution to map out the grain structure as well as good resolution of the orientation and strain data. The employed beam height of $100 \mu \mathrm{m}$ and the subsequent data analysis algorithms are optimised to provide information at the level of individual grains in terms of grain averaged orientations and elastic strains. Nevertheless, the data contain more detailed information on the orientation spread within a grain. Alternative experimental set ups may provide higher spatial resolution and thus more detailed analysis of the orientation spread, e.g. [6,25,26]. The present data have been collected only at a few strain levels. Application of smaller strain steps allows detection of specific phenomena, e.g. deformation twinning $[27,28]$. Faster detectors also open up for studies of e.g. relaxation phenomena [29].

At the atomic level, plastic deformation of metals occur by dislocation glide and multiplication. It is well-known that the dislocations self-organise into boundaries of different character, a process that is not yet well-understood. The grain orientation - or more precisely the combination of active slip systems - has been determined to be an important factor controlling the type of boundaries formed, in particular their crystallographic alignment [30-33]. Transmission electron microscopy has also established that the dislocations in the boundaries come from the active slip system [3436]. Analysis of electron microscopy data reveals that the dislocations in the boundaries to a large extent screen each others' stress fields [35-37], although local stress data from synchrotron experiments show that the screening is not perfect [38]. The mechanisms of the self-organisation is, however, mostly studied by dislocation dynamics simulations due to the lack of experimental data. New emerging synchrotron techniques [39-41] will in the near future provide experimental data on this scientifically intriguing self-organisation process and the dislocation interactions that lead to the technologically important work hardening of metals.

\section{Conclusions}

Data from a single 3DXRD experiment on the tensile deformation of austenitic stainless steel (316L) have been analysed, leading to the following conclusions

- The elastic interaction across twin boundaries. In spite of the fact that these boundaries involve interaction in the elastically stiffest direction in the fcc crystal lattice, no special correlation between the stress normal to the boundary in the two parts of the twin was found. This means that in the modelling of grain averaged stresses, twin boundaries can safely be treated as conventional grain boundaries.

- The stress state of individual grains. In the elastic regime this state appears dominated by the applied uniaxial stress. In the plastic regime about half of the grains enter a Bishop-Hill stress state. The orientation dependence of these stress states deviates from the expected. This is attributed to grains not deforming with axisymmetric strains. Crystal plasticity simulations reproduce the overall trends, but fail at the level of individual grains.

- The rotation paths of individual grains exhibit more scatter than predicted by crystal plasticity models. The rotation angles are also larger. 
Based on the present results it must be concluded that the combination of grain-resolved experimental data and crystal plasticity simulations is essential to provide the new insight necessary to improve our predictive capacity.

\section{Acknowledgements}

This work is based upon research conducted at the Cornell High Energy Synchrotron Source (CHESS), which is supported by the NSF \& NIH/NIGMS via NSF award DMR-1332208. The authors are grateful to Drs. Paul Shade, Margaret K. A. Koker and Darren Dale for collaboration on the synchrotron experiment. The Danish Independent Research Council - Technology and Productions Sciences grant n. DFF-13555-00220 and the Danish Agency for Science, Technology and Innovation (through Danscatt) are acknowledged for financial support.

\section{References}

[1] H.F. Poulsen, S.F. Nielsen, E.M. Lauridsen, S. Schmidt, R.M. Suter, J. Appl. Crystallogr.,34, 751 (2001).

[2] U. Lienert, S.F. Li, C.M. Hefferan, J. Lind, R.M. Suter, J. V. Bernier, N.R. Barton, M.C. Brandes, M.J. Mills, M.P. Miller, B. Jakobsen, W. Pantleon, JOM, 63, 70 (2011).

[3] S.E. Offerman, N.H. van Dijk, J. Sietsma, S. Grigull, E.M. Lauridsen, L. Margulies, H.F. Poulsen, M.T. Rekveldt, S. van der Zwaag, Science, 298, 1003 (2002)

[4] S. Schmidt, S. Nielsen, C. Gundlach, L. Margulies, X. Huang, D. Juul Jensen, Science, 305, 229 (2004).

[5] S. Schmidt, U.L. Olsen, H.F. Poulsen, H.O. Sorensen, E.M. Lauridsen, L. Margulies, C. Maurice, D.J. Jensen, Scr. Mater., 59, 491 (2008).

[6] S.F. Li, J. Lind, C.M. Hefferan, R. Pokharel, U. Lienert, A.D. Rollett, R.M. Suter, J. Appl. Crystallogr. ,45, 1098 (2012).

[7] B. Jakobsen, H.F. Poulsen, U. Lienert, J. Almer, S.D. Shastri, H.O. Sørensen, C. Gundlach, W. Pantleon, Science 312, 889 (2006).

[8] C. Wejdemann, H.F. Poulsen, U. Lienert, W. Pantleon, J. Microsc., 65, 35 (2013).

[9] J. V. Bernier, N.R. Barton, U. Lienert, M.P. Miller, J. Strain Anal. Eng. Des., 46, 527 (2011). 
[10] J.H. Lee, C.C. Aydiner, J.D. Almer, J.V. Bernier, K.W. Chapman, P.J. Chupas, D.R. Haeffner, K. Kump, P.L. Lee, U. Lienert, A. Miceli, G. Vera, J. Synchrotron Radiat., 15, 477 (2008)

[11] P.A. Shade, B. Blank, J.C. Schuren, T.J. Turner, P. Kenesei, K. Goetze, R.M. Suter, J. V. Bernier, S.F. Li, J. Lind, U. Lienert, J. Almer, Rev. Sci. Instrum., 86, 093902 (2015).

[12] H.O. Sørensen, S. Schmidt, J.P. Wright, G.B.M. Vaughan, S. Techert, E.F. Garman, J. Oddershede, J. Davaasambu, K.S. Paithankar, C. Gundlach, H.F. Poulsen, Zeitschrift Fur Krist., 227, 63 (2012).

[13] S. Schmidt, J. Appl. Crystallogr., 47, 276 (2014)

[14] J. Oddershede, S. Schmidt, H.F. Poulsen, H.O. Sørensen, J. Wright, W. Reimers, J. Appl. Crystallogr., 43, 539 (2010).

[15] N.Y. Juul, G. Winther, D. Dale, M.K.A. Koker, P. Shade, J. Oddershede, Scr. Mater., 120, 1 (2016).

[16] H.M. Ledbetter, Br. J. Non-Destructive Test., 23, 286 (1981).

[17] N.Y. Juul, J. Oddershede, A. Beaudoin, K. Chatterjee, M.K.A. Koker, D. Dale, P. Shade, G. Winther, Acta Mater., 141, 388 (2017).

[18] K. Chatterjee, A. Venkataraman, T. Garbaciak, J. Rotella, M.D. Sangid, A. Beaudoin, Int. J. Solids Struct., 94-95, 1 (2015).

[19] J. Bishop, R. Hill, Philos. Mag., 42, 414 (1951).

[20] B. Clausen, T. Leffers, T. Lorentzen, O.B. Pedersen, P. Van Houtte, Scr. Mater., 42, 91 (1999).

[21] G. Winther, L. Margulies, S. Schmidt, H.F. Poulsen, Acta Mater., 52, 2863 (2004).

[22] M. Messner, A. Beaudoin, R. Dodds, Eng. Comput., 32, 1526 (2015).

[23] R. Pokharel, J. Lind, S. Fai, P. Kenesei, R. Lebensohn, R.M. Suter, A.D. Rollett, Int. J. Plast., 67, 217 (2015).

[24] J. Oddershede, J.P. Wright, A. Beaudoin, G. Winther, Acta Mater., 85, 301 (2015).

[25] G. Winther, J.P. Wright, S. Schmidt, J. Oddershede, Int. J. Plast., 88, 108 (2017). 
[26] R. Pokharel, R.A. Lebensohn, Scr. Mater., 132, 73 (2017).

[27] T.R. Bieler, L. Wang, A.J. Beaudoin, P. Kenesei, U. Lienert, Metall. Mater. Trans. A., 45, 109 (2014).

[28] C.C. Aydiner, J. V. Bernier, B. Clausen, U. Lienert, C.N. Tomé, D.W. Brown, Phys. Rev. B - Condens. Matter Mater. Phys., 80, 1 (2009).

[29] K. Chatterjee, A.J. Beaudoin, D.C. Pagan, P.A. Shade, H.T. Phillipp, M.W. Tate, S.M. Gruner, P. Kenesei, J.-S. Park, Struct. Dyn., 6, 014501 (2019).

[30] G. Le, A. Godfrey, N. Hansen, W. Liu, G. Winther, X. Huang, Acta Mater., 61, 7072 (2013).

[31] N. Hansen, X. Huang, W. Pantleon, G. Winther, Philos. Mag., 86, 3981 (2006).

[32] G.M. Le, A. Godfrey, C.S. Hong, X. Huang, G. Winther, Scr. Mater., 66, 359 (2012).

[33] G. Winther, Scr. Mater., 52, 995 (2005).

[34] C.S. Hong, X. Huang, G. Winther, Philos. Mag., 93, 3118 (2013).

[35] Y. Wei, A. Godfrey, W. Liu, Q. Liu, X. Huang, N. Hansen, G. Winther, Scr. Mater., 65355 (2011).

[36] R.J. McCabe, A. Misra, T.E. Mitchell, Acta Mater., 52, 705 (2004).

[37] G. Winther, C.S. Hong, X. Huang, Philos. Mag., 95, 1471 (2015).

[38] I.-F. Lee, T.Q. Phan, L.E. Levine, J.Z. Tischler, P.T. Geantil, Y. Huang, T.G. Langdon, M.E. Kassner, Acta Mater., 61, 7741 (2013).

[39] H. Simons, A.C. Jakobsen, S.R. Ahl, C. Detlefs, H.F. Poulsen, Multiscale 3D characterization with dark-field x-ray microscopy, MRS Bull., 41, 454 (2016).

[40] S.R. Ahl, H. Simons, Y.B. Zhang, C. Detlefs, F. Stöhr, A.C. Jakobsen, D. Juul Jensen, H.F. Poulsen, Scr. Mater., 139, 87 (2017).

[41] J.X. Sierra, H.F. Poulsen, P.S. Jørgensen, C. Detlefs, P. Cook, H. Simons, A.C. Jakobsen, J.R. Bowen, J. Power Sources., 413, 351 (2019). 


\section{Figures}
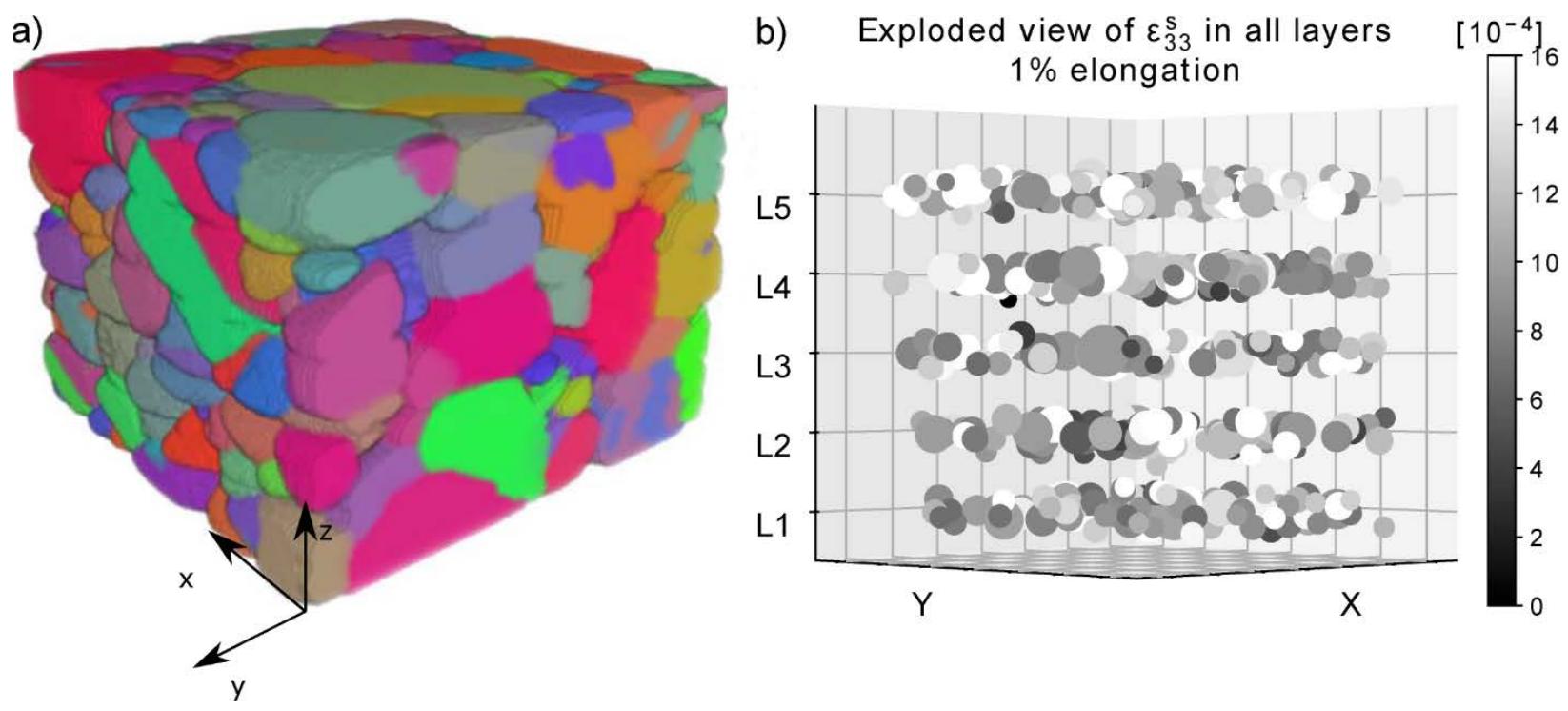

Fig. 1. a) Measured space-filling grain map in the undeformed state $\left(0.7 \times 0.7 \times 0.5 \mathrm{~mm}^{3}\right)$. Colours mark crystallographic orientations at an arbitrary scale. b) The tensile elastic strain measured for individual grains in the 5 consecutive layers of $100 \mu \mathrm{m}$ height at $1 \%$ elongation. The size of the spheres reflects the relative grain volumes (scaled down for clarity), the position is the centre-ofmass position and colours represent the average tensile elastic strain in the grain. 

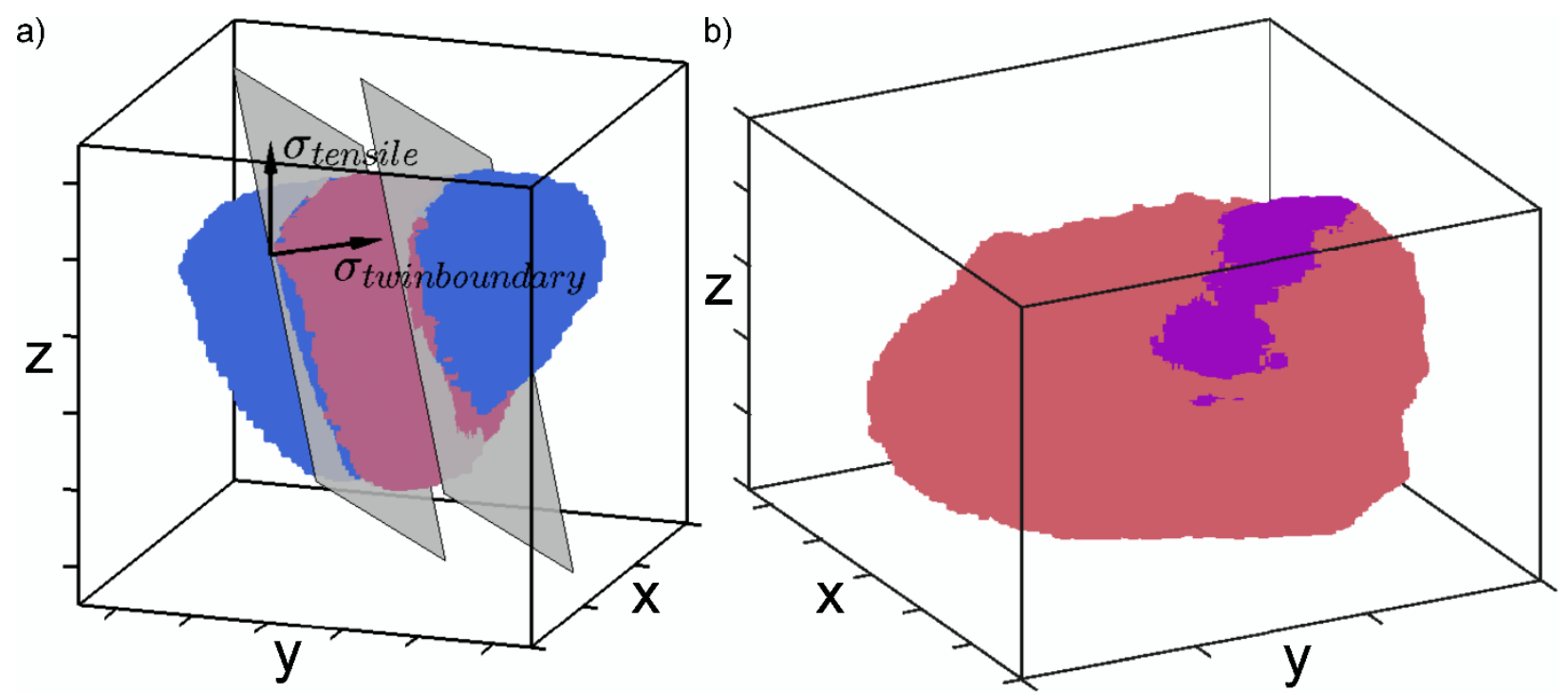

Fig. 2. Two grains with annealing twins extracted from the experimental grain map in Fig. 1a (enclosing box of a) $0.15 \times 0.15 \times 0.15 \mathrm{~mm}^{3}$ and b) $0.30 \times 0.30 \times 0.30 \mathrm{~mm}^{3}$ ).
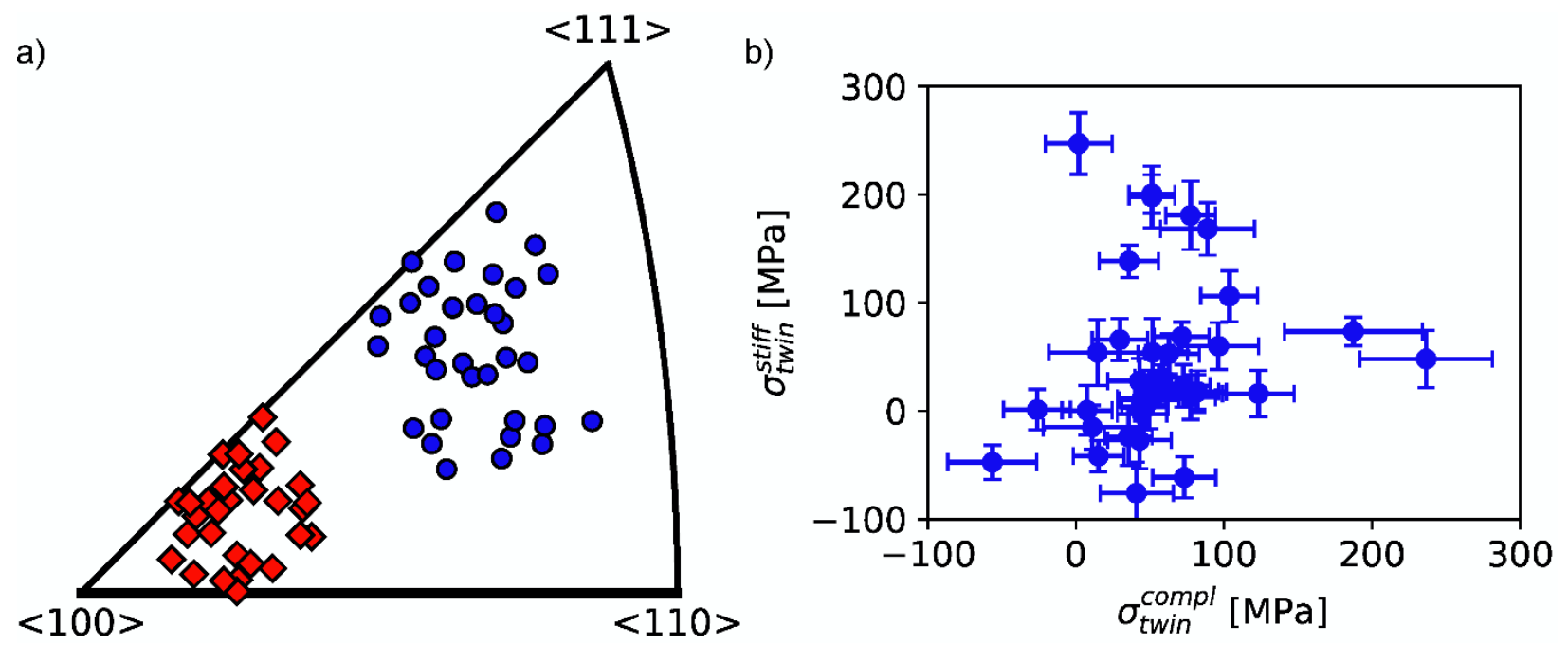

Fig. 3. a) Crystallographic orientations of the tensile axes of the compliant and stiff parts of the selected twin pairs represented by red diamonds and blue circles, respectively. b) The two stress components normal to the twin boundary for each twin pair. Data from [15]. 


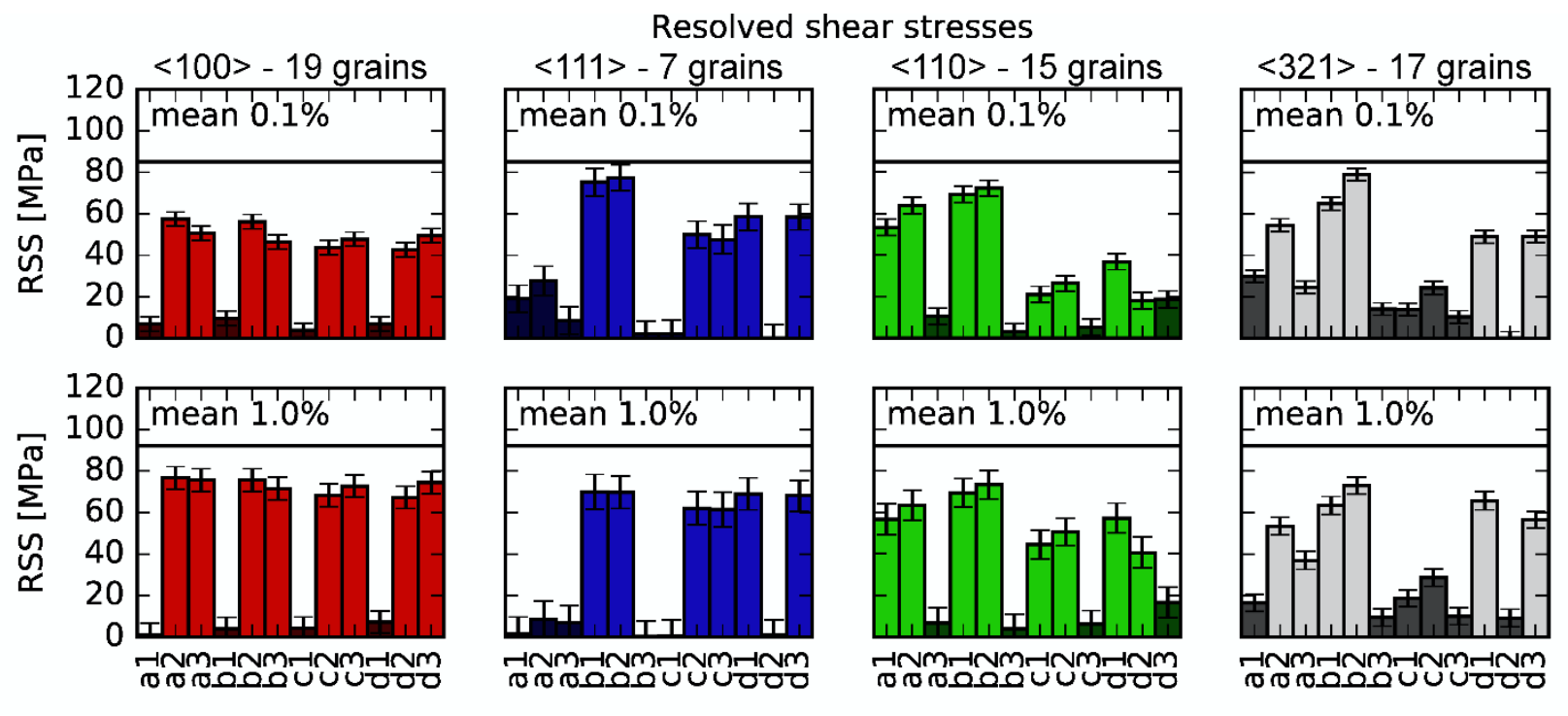

Fig. 4 . Resolved shear stresses averaged over grains with tensile axes in the immediate vicinity of $<100>,<111>,<110>$ and $<321>$. Grain orientations are converted to the same stereographic triangle where slip system b2 has the highest Schmid factor in uniaxial tension. Highlighted colours mark the slip systems of the Bishop-Hill stress state expected for the specific orientation. The horizontal line marks the expected critical resolved shear stress. Top row) $0.1 \%$ elongation; Bottom row) 1\% elongation. 


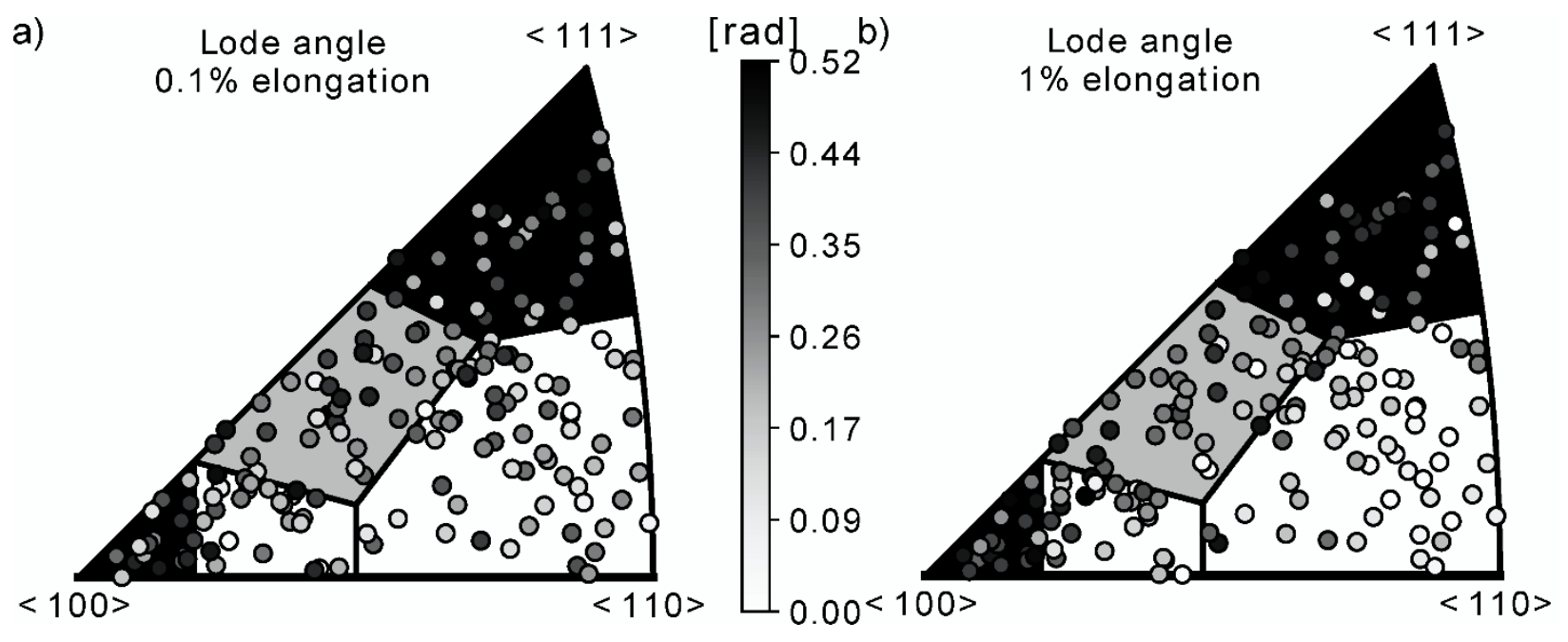

Fig. 5. Stereographic triangles presenting the orientation dependence of the five Bishop-Hill stress states in the form of the Lode angle as background colours. The symbols mark the orientation of the tensile axis of the individual grains, colour coded according to their Lode angles.

a)

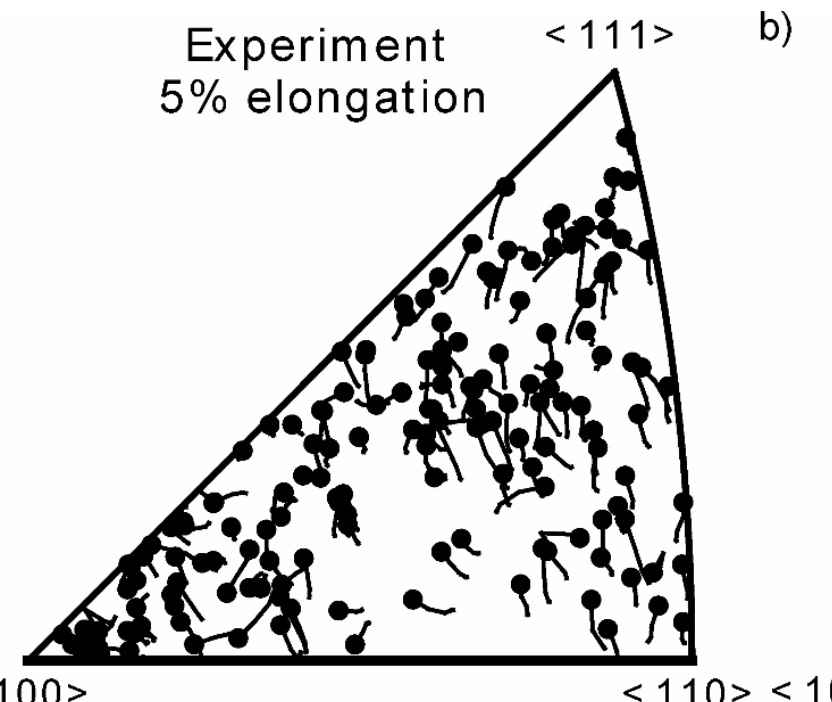

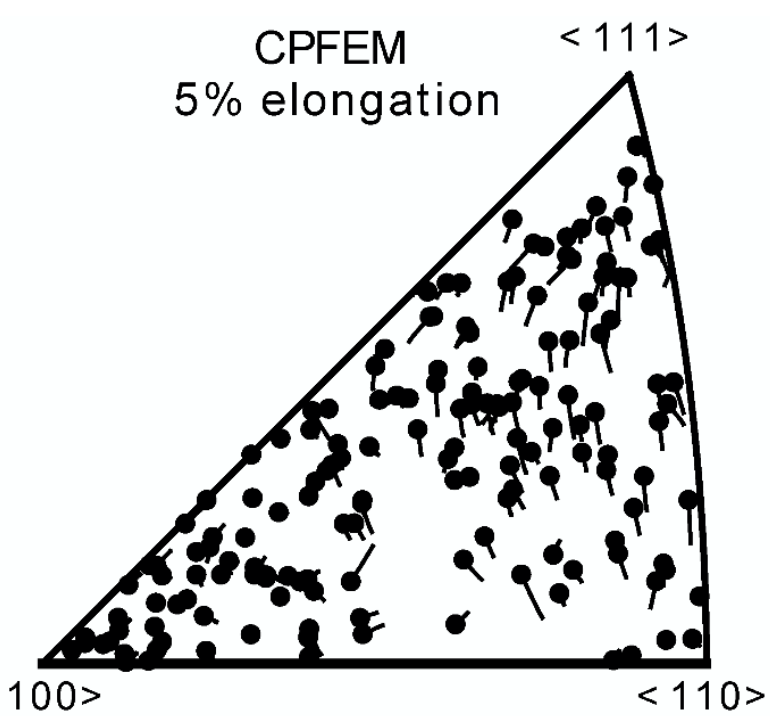

Fig. 6. Stereographic triangles showing the CMS rotations from 0 to $5 \%$ elongation. a) experimental rotation paths; b) predicted rotation paths using the WARP3D crystal plasticity software. The symbols mark the final orientation of the tensile axis. 


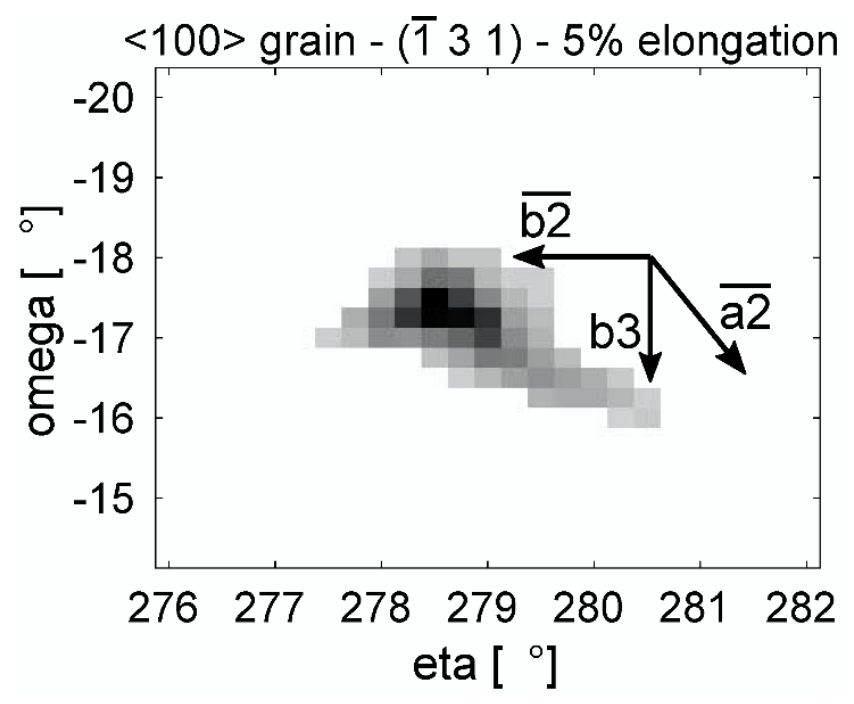

Fig. 7. Experimental diffraction intensity integrated over $2 \theta$ and presented as a function of the angles $\eta$ (marking the position on the Debye-Scherrer ring on the detector) and $\omega$ (marking rotation around the tensile axis). The arbitrary colour scale goes from the blue background value over green to yellow at the maximum intensity. Arrows indicate the rotation directions caused by individual slip systems. 
Table I. Grain resolved mean error bars of the stress tensor of about 350 grains. The tensile direction is along the 3 -axis.

\begin{tabular}{|c|c|c|c|}
\hline \multirow{2}{*}{$\begin{array}{l}\text { Elongation } \\
\text { [\%] }\end{array}$} & \multicolumn{3}{|c|}{ Grain-resolved error bars } \\
\hline & $\begin{array}{l}\sigma_{33} \\
{[\mathrm{MPa}]}\end{array}$ & $\begin{array}{l}\left(\sigma_{\left.11+\sigma_{22}\right) / 2}\right. \\
{[\mathrm{MPa}]}\end{array}$ & $\begin{array}{l}\left(\sigma_{12+} \sigma_{13+} \sigma_{23}\right) / 3 \\
{[\mathrm{MPa}]}\end{array}$ \\
\hline 0.1 & 14 & 21 & 8 \\
\hline 1 & 15 & 22 & 9 \\
\hline
\end{tabular}

Table II. Slip systems in Bishop-Hill notation

\begin{tabular}{|c|c|c|c|c|}
\hline Plane & (111) & $(\overline{1} \overline{1} 1)$ & $(\overline{1} 11)$ & $(1 \overline{1} 1)$ \\
\hline Direction & {$[01 \overline{1}][\overline{1} 01][1 \overline{1} 0]$} & {$[0 \overline{1} \overline{1}][101][\overline{1} 10]$} & {$[01 \overline{1}][101][\overline{1} \overline{1} 0]$} & {$[0 \overline{1} \overline{1}][\overline{1} 01][110]$} \\
\hline Notation & a2 a3 & b2 b3 & c2 & $\mathrm{d} 2 \quad \mathrm{~d} 3$ \\
\hline
\end{tabular}

